\title{
Redistribution of myocardial perfusion during permanent dual chamber pacing in symptomatic non-obstructive hypertrophic cardiomyopathy: a quantitative positron emission tomography study
}

\author{
Jan L Posma, Paul K Blanksma, Ernst E van der Wall
}

\begin{abstract}
Dual chamber pacing causes significant symptomatic improvement in many patients with hypertrophic cardiomyopathy. The mechanism behind this beneficial response is not fully understood.

Positron emission tomography showed a redistribution of myocardial flow during pacing in a patient with non-obstructive hypertrophic cardiomyopathy. Early septal activation reduced septal fibre strain and blood flow and increased septal perfusion reserve.
\end{abstract}

(Heart 1996;75:522-524)

Keywords: hypertrophic cardiomyopathy; pacing; perfusion imaging

Permanent dual chamber pacing in obstructive hypertrophic cardiomyopathy is highly effective in relieving symptoms, improving exercise performance, and reducing left ventricular outflow tract obstruction. ${ }^{1}$ In patients with obstructive hypertrophic cardiomyopathy dual chamber pacing improves myocardial perfusion, as demonstrated by exercise thallium-201 scintigraphy. ${ }^{2}$ Also in patients with non-obstructive hypertrophic cardiomyopathy chronic dual chamber pacing is associated with improvement in symptoms and exercise tolerance, ${ }^{34}$ but the mechanism underlying this benefit is not clear. We recently encountered a patient with hypertrophic cardiomyopathy without obstruction of the left ventricular outflow tract in whom dynamic positron emission tomography showed extensive abnormalities in myocardial perfusion, which normalised during dual chamber pacing.

\section{Case report}

A 55 year old man was known to have had hypertrophic cardiomyopathy for 25 years. There was obstruction of the left ventricular outflow tract (gradient $100 \mathrm{~mm} \mathrm{Hg}$ at rest). Septal wall thickness was $23 \mathrm{~mm}$ and lateral wall thickness was $11 \mathrm{~mm}$. Nine years before a septal myectomy had been performed, because he presented with severe limiting symptoms of angina unresponsive to medication. Despite a successful procedure his symptoms did not improve. Over the years his symptoms progressively worsened to New York Heart Association functional class IV and he was admitted to our hospital. During a symptom limited bicycle exercise test he exercised to $90 \mathrm{~W}$ and stopped because of chest pain. Cardiac catheterisation did not demonstrate significant outflow tract obstruction at rest or after provocation procedures, and showed normal coronary arteries without systolic compression. Left ventriculography showed cavity obliteration and a thickened akinetic interventricular septum. Mitral or aortic regurgitation were not present. Thallium-201 scintigraphy showed reversible perfusion defects suggestive of septal wall ischaemia.

A dual chamber pacemaker was inserted (Intermedics Relay). Ventricular capture was maintained at maximum exercise by programming the atrioventricular delay to $110 \mathrm{~ms}$ and activating the rate adaptive atrioventricular interval shortening. With dual chamber pacing the functional state of the patient improved from NYHA class IV to class II. He was able to exercise on a bicycle for longer during pacing therapy than he could during sinus rhythm before pacemaker implantation: $8 \mathrm{~min}$ (110 W) $v 5 \mathrm{~min}(90 \mathrm{~W})$. The left ventricular end diastolic and end systolic dimensions were unchanged during pacing (36 and $22 \mathrm{~mm}$ ). The thickness of the septal and lateral walls did not change during three months follow up.

Before and three months after pacemaker insertion, regional myocardial perfusion was assessed quantitatively with dynamic positron emission tomography using an ECAT Siemens 951/31 tomographic system. Rest and stress dipyridamole perfusion imaging with ${ }^{13} \mathrm{~N}$ ammonia was performed during regular sinus rhythm, and during dual chamber pacing. All medication was stopped two days before the positron emission tomography procedure. Dynamic image acquisition, data analysis, and parametric polar map display were obtained as described earlier. ${ }^{5}$ Briefly, data were reorientated into 10 left ventricular short axis slices. In each slice the myocardium was divided into 48 segments. For all 480 segments time activity curves were assessed using maximum activity. 

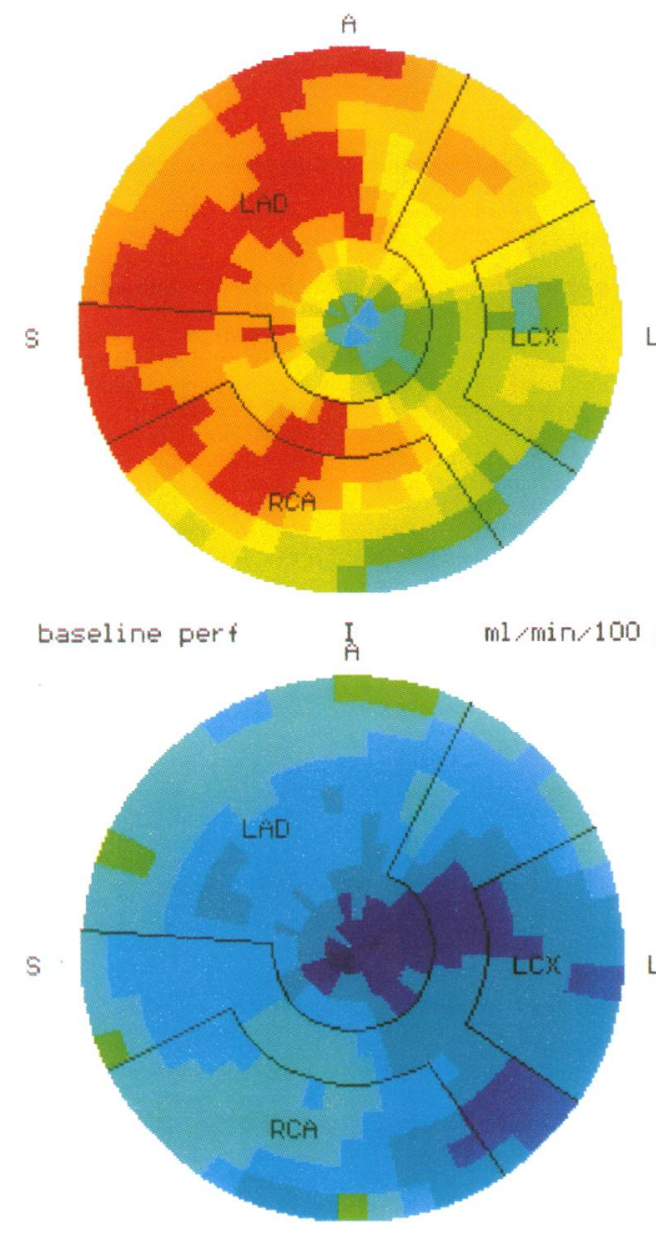

pacing pertusion
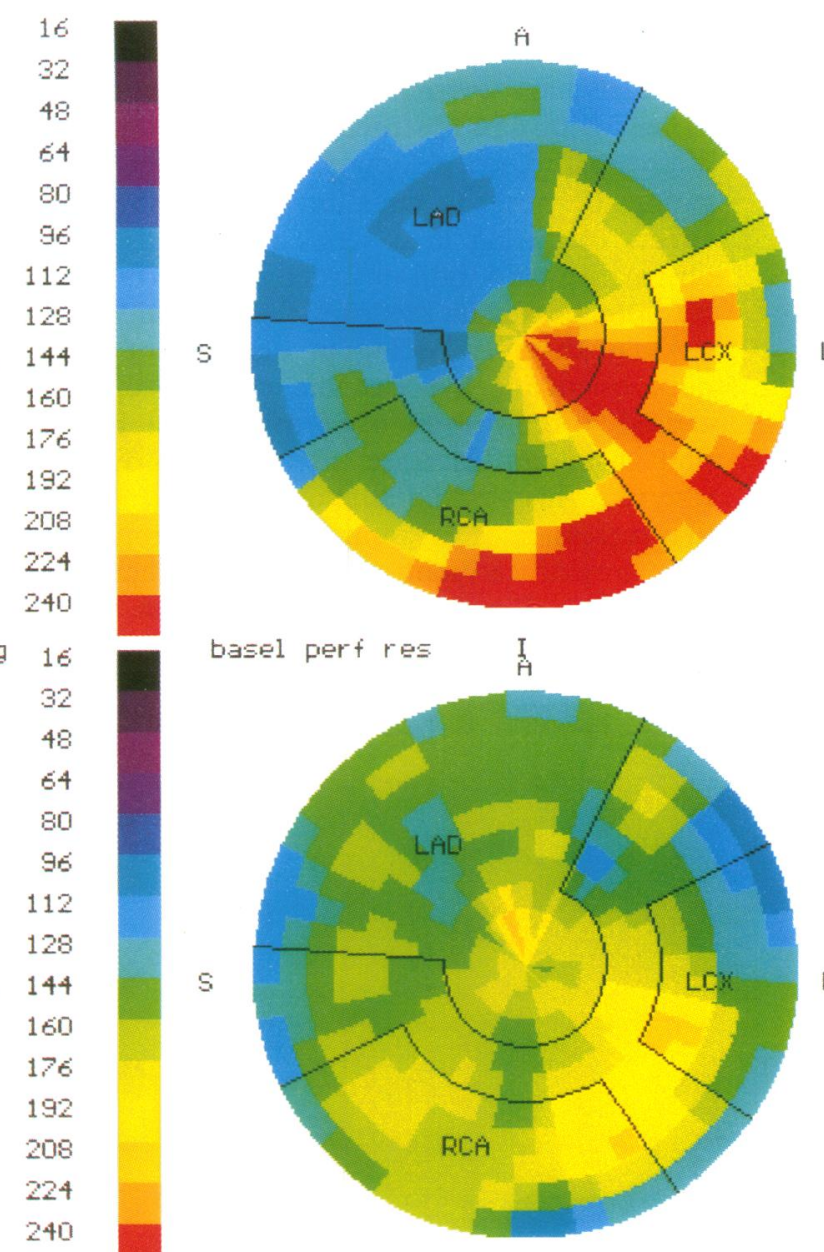

pacing perf reserweI

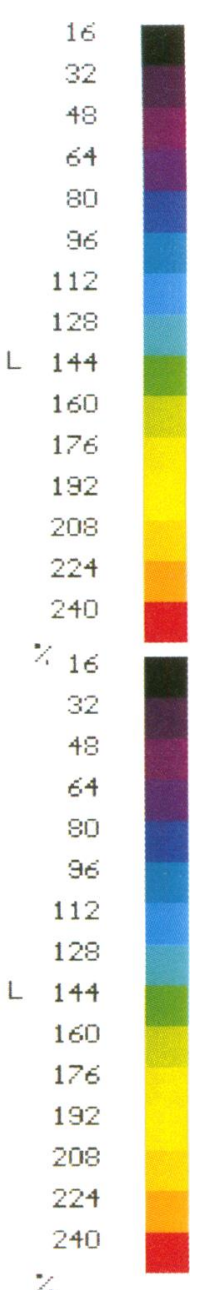

Parametric polar maps of dynamic positron emission tomography studies before (upper panels) and after pacemaker implantation (lower panels). The ${ }^{13} \mathrm{~N}-$ ammonia image during sinus rhythm (left upper) shows high myocardial perfusion in the anteroseptal region with a low anteroseptal perfusion reserve during dipyridamole stress (right upper). During dual chamber pacing resting perfusion was lower in all regions (left lower), with a homogeneous distribution of perfusion reserve (right lower).

In each segment absolute myocardial perfusion at rest and after dipyridamole were assessed and displayed in polar maps. Myocardial perfusion reserve was defined as the ratio of dipyridamole stimulated perfusion to resting perfusion. Data were not corrected for partial volume effect, because the developed correction models might not be appropriate for hypertrophic cardiomyopathy with complex motion of the heart and irregular distribution of hypertrophy. Furthermore, in our case correction was not necessary because results within one patient were being compared.

During sinus rhythm, resting myocardial perfusion was irregularly distributed, and anterior and septal flow reserve were diminished. During dual chamber pacing, resting perfusion was lower in all segments, and perfusion reserve

Myocardial perfusion and perfusion reserve during sinus rhythm and during dual chamber pacing

\begin{tabular}{lll}
\hline & $\begin{array}{l}\text { Rest perfusion } \\
\text { (ml/100g/min) }\end{array}$ & $\begin{array}{l}\text { Perfusion } \\
\text { reserve }\end{array}$ \\
\hline Sinus rhythm: & 194 & 1.66 \\
Anterior & 210 & 1.20 \\
Septal & 225 & 1.19 \\
Inferior & 180 & 2.20 \\
Lateral & 160 & 2.06 \\
Dual chamber pacing: & 107 & 1.58 \\
Anterior & 110 & 1.45 \\
Septal & 112 & 1.50 \\
Inferior & 116 & 1.65 \\
Lateral & 88 & 1.72 \\
\hline
\end{tabular}

was more homogeneously distributed than baseline measurements (figure and table). The double product at rest and after dipyridamole stress did not differ between sinus rhythm and pacing studies.

\section{Discussion}

The present findings indicate that dual chamber pacing might provide symptomatic improvement in selected patients with nonobstructive hypertrophic cardiomyopathy. At present the mechanisms of improvement are unclear. The improvement after a longer period of dual chamber pacing in obstructive hypertrophic cardiomyopathy ${ }^{1}$ and the sustained improvement after temporary cessation of pacing suggest adaptive cardiac changes. The increased basal blood flow and decreased flow reserve of the interventricular septum may be the result of isometric fibre contraction in areas with myocardial fibre disarray. Pacing causes an asynchronous pattern of ventricular activation. In animal studies, pacing has been shown to cause segmental differences in contractile work and to induce a decrease of myocardial oxygen consumption in regions that are activated early. ${ }^{6}$ Early septal activation by right ventricular pacing has been shown to reduce septal fibre strain and blood flow. This accords with our positron emission tomography findings which 
showed redistribution of myocardial flow during dual chamber pacing. After this finding in a patient with non-obstructive hypertrophic cardiomyopathy, we are now prospectively investigating, by positron emission tomography, the effects of pacing in a series of patients with obstructive and non-obstructive hypertrophic cardiomyopathy on myocardial perfusion.

Dual chamber pacing in our patient with non-obstructive hypertrophic cardiomyopathy caused a redistribution of myocardial blood flow with an increase in septal perfusion reserve. This finding supports the hypothesis that regression of left ventricular hypertrophy in hypertrophic cardiomyopathy during pacing is caused by a decrease in septal fibre strain.

1 Fananapazir I Epstein ND, Curiel RV, Panza JA, Tripody $D$, McAreavey $D$. Long-term results of dual-chamber
(DDD) pacing in obstructive hypertrophic cardiomyopathy, evidence for progressive symptomatic and hemodynamic improvement and reduction of left

2 Fananapazir L, Dilsizian V, Bonow RO. Dual chamber pacing relieves angina and improves myocardial perfusion abnormalities in patients with obstructive hypertrophic cardiomyopathy (abstract). Circulation 1992;86

3 Seidelin PH, Jones GA, Boon NA. Effects of dual-chamber pacing in hypertrophic cardiomyopathy without obstruction (letter). Lancet 1992;340:369.

4 Cannon RO III, Tripodi D, Dilsizian V, Panza JA Fananapazir L. Results of permanent dual-chamber pacing in symptomatic nonobstructive hypertrophic cardiomyopathy. Am f Cardiol 1994;73:571-6.

5 Blanksma PK, Willemsen ATM, Meeder JG, et al. Quantitative myocardial mapping of perfusion and metabolism using parametric polar map displays in cardiac pet. $7 \mathrm{Nucl}$ Med 1995;36:153-8.

6 Delhaas T, Arts T, Prinzen FW, Reneman RS. Relation between regional electrical activation time and subepicardial fiber strain in the canine left ventricle. Pflügers Arch (Eur f Physiol) 1993;423:78-87.

7 Prinzen FW, Augustijn CH, Arts T, Allessie MA, Reneman RS. Redistribution of myocardial fiber strain and blood flow by asynchronous activation. Am $\mathcal{F}$ Physiol 1990;259: H300-8. 